\title{
PRIME QUADRATICS ASSOCIATED WITH COMPLEX QUADRATIC FIELDS OF CLASS NUMBER TWO
}

\author{
M. D. HENDY
}

\begin{abstract}
We establish a necessary and sufficient relation between those quadratic fields of class number two, and some quadratic polynomials $f(x)$ which take only prime values for small positive integers.
\end{abstract}

Euler discovered that for certain primes $q$, namely $q=2,3,5,11,17,41$, the quadratic

$$
f(x)=x^{2}+x+q
$$

takes only prime values for integers in the interval $0 \leqq x \leqq q-2$. (Cf. [1].) In fact it is known that a prime $q$ is such a value if and only if the complex quadratic field $Q(\sqrt{ }(1-4 q))$ has class number one. This test readily gives rise to all the fields $Q(\sqrt{ }-d)$ with $d \geqq 7$, which have class number one. In a similar manner we discover a quadratic similar to (1) related to each of the complex quadratic fields of class number two.

Let $d$ be any squarefree positive integer, and $h$ be the class number of the field $Q(\sqrt{ }-d)$. This field has discriminant $D=-d$ when $d \equiv 3(\bmod 4)$, or $D=-4 d$ otherwise. From the theory of genera of complex quadratic fields, we note that the class group of $Q(\sqrt{ }-d)$ will contain one factor of order a power of two if and only if $D$ has precisely two distinct prime factors. Hence $h$ can only be two for fields $Q(\sqrt{ }-d)$ of one of the following three types:

I. $d=2 p, p$ odd prime, $D=-8 p$.

II. $d=p \equiv 1(\bmod 4), p$ prime,$D=-4 p$.

III. $d=p q \equiv 3(\bmod 4), p, q$ prime,$D=-p q$.

For fields of Type III, we will assume that $p<q$. For each field we associate a quadratic $f(x)$ similar to (1). For fields of Type $\mathrm{I}$,

$$
f(x)=2 x^{2}+p .
$$

Received by the editors June 7, 1971 and, in revised form, February 14, 1972 and July 30, 1973.

AMS (MOS) subject classifications (1970). Primary 12A25, 12A50; Secondary $10 \mathrm{H} 15$.

Key words and phrases. Complex quadratic fields, class number, prime integers, ideals, principal ideals.

(c) American Mathematical Society 1974 
For fields of Type II,

$$
f(x)=2 x^{2}+2 x+(p+1) / 2 .
$$

For fields of Type III,

$$
f(x)=p x^{2}+p x+(p+q) / 4 .
$$

THEOREM. A complex qua ratic field of Type I, II or III has class number $h=2$ if and only if the corresponding quadratic $f(x)$ takes only prime values for integers $x$ in the interval $0 \leqq x<k$, where $k=\sqrt{ }(p / 2)$ for fields of Type I, $k=(\sqrt{ } p-1) / 2$ for fields of Type II, ana $k=\sqrt{ }(p q / 12)-\frac{1}{2}$ for fields of Type III.

Proof. The proof is established via the following lemmas.

LEMMA 1. If $x$ is the least positive integer for which $f(x)=2 x^{2}+p$ of (2) is composite, then $x<(p-1) / \sqrt{ } 2 \Rightarrow x<\sqrt{ }(p / 2)$.

Proof. $f(p)$ is composite, so a minimal positive integer $x$ for which $f(x)$ is composite does exist. Set $L_{0}=(p-1) / \sqrt{ } 2, L=\sqrt{ }(p / 2)$ and $L_{n}=\sqrt{ }\left(L_{0}^{2} 2^{-n}+\left(2^{n}-1\right) p 2^{-n-1}\right)$, and suppose $x<L_{0}$. We find, for $n \geqq 0$,

$$
\begin{gathered}
L<L_{n+1}<L_{n} \leqq L_{0}, \\
L=\lim _{n \rightarrow \infty} L_{n} \text { and } \\
L_{n+1}=\frac{1}{2} \sqrt{ }\left(2 L_{n}^{2}+p\right) .
\end{gathered}
$$

Suppose, for some $n \geqq 0, x<L_{n}$. As $f(x)$ is composite let $a$ be its least prime divisor. Thus

$$
a^{2} \leqq f(x)<2 L_{n}^{2}+p \leqq 2 L_{0}^{2}+p=(p-1)^{2}+p<p^{2},
$$

and, in particular,

$$
a<\sqrt{ }\left(2 L_{n}^{2}+p\right)<p .
$$

Thus $f(y)=a$ has no real roots. Now

$$
f(|x-a|) \equiv f(x) \equiv 0(\bmod a),
$$

so that $f(|x-a|)$ also has $a$ as a proper divisor, and hence is composite. $f(0)=p$, so $x \neq a$, and as $x$ is minimal $x<|x-a|$, i.e.,

$$
x \leqq a / 2<\frac{1}{2} \sqrt{ }\left(L_{n}^{2}+p\right)=L_{n+1} .
$$

Thus by induction $x<L_{0} \Rightarrow x<L_{n}$ for each $n \geqq 0$, and so $x \leqq \lim _{n \rightarrow \infty} L_{n}=$ $L$. Equality cannot hold as $L$ is irrational, so $x<L$ establishing the lemma. 
Lemma 2. If $x$ is the least positive integer for which $f(x)=2 x^{2}+2 x+$ $(p+1) / 2$ of (3) is composite, then $x<(p-\sqrt{ } 2) / 2 \sqrt{ } 2 \Rightarrow x<(\sqrt{ } p-1) / 2$.

Proof. We use the same procedure as above in Lemma 1 , with $L=(\sqrt{ } p-1) / 2, L_{0}=(p-\sqrt{ } 2) / 2 \sqrt{ } 2$ and

$$
L_{n}=\sqrt{ }\left(\left(L_{0}+\frac{1}{2}\right)^{2} 2^{-n}+\left(2^{n}-1\right) p 2^{-n-2}\right)-\frac{1}{2} .
$$

In equation (10) we replace $f(x-a) \equiv f(a-x) \equiv f(x) \equiv 0(\bmod a)$ with $f(x-a) \equiv f(a-x-1) \equiv f(x) \equiv 0(\bmod a)$, so for $x$ minimal, $x<a$ and $x<a-x-1$. The remainder of the proof then follows.

Unfortunately a corresponding result for $f(x)=p x^{2}+p x+(p+q) / 4$ of (4) does not exist for $p>3$. For fields of Types I and II set $a=2$ and for fields of Type III set $a=p$. Let $A$ be the ambiguous ideal with $N(A)=a$. This meaning of the letter $a$ has nothing to do with its use in Lemmas 1 and 2. From now on $a$ will have the meaning specified here.

Lemma 3. If $h>2$, then there exist nonprincipal ideals $B, C$ with the following properties:

1. $B$ and $C$ are neither principal nor in the same class as $A$.

2. $A B C$ is principal.

3. $B$ is a prime ideal.

4. $1<N(B), N(C)<\sqrt{ }(-D / 3)$.

5. $A \nmid B C$.

Proof. As $N(A)<-D / 4, A$ cannot be principal. Let $K_{1}$ be the class of principal ideals and $K_{2}$ the class containing $A$. As $h>2$ and $K_{2}^{2}=K_{1}$, $\left\{K_{1}, K_{2}\right\}$ is a proper subgroup of the class group. Hence there exist other classes, and at least one of them, say $K_{3}$, has a prime ideal $B$ as its member of least norm. As $K_{3}$ is distinct from $K_{1}, K_{2}$, so too is $K_{4}=K_{2} K_{3}^{-1}$. Let $C$ be an ideal of least norm of $K_{4}$, so that $A B C \in K_{2} K_{3} K_{4}=K_{2}^{2}=K_{1}$ is a principal ideal.

We have an upper bound (see [1, p. 141]) on the size of the least (according to norm) ideal of any equivalence class $K_{i}$. That is, in each class $K_{i}$ over $Q(\sqrt{ }-d)$ there exists an ideal $A_{i}$, with $N\left(A_{i}\right)<\sqrt{ }(-D / 3)$. Hence $B, C$ are ideals satisfying properties $1,2,3$ and 4 .

Further $A|B C \Rightarrow A| C$ as $B$ is prime, so that there would need to exist an ideal $E$, with $C=A E$. This would mean $B E \sim A^{2} B E=A B C$, so $B E$ is principal; however, as $N(E) \leqq N(C) / 2<\frac{1}{2} \sqrt{ }(-D / 3), N(B)<\sqrt{ }(-D / 3)$, and $N(B E)<-D / 6$ it could not be principal. Hence $A \nmid B C$.

LEMmA 4. In the fields of Type I, $h>2 \Rightarrow f(x)$ is composite for some integer $x$ in the interval $0 \leqq x<\sqrt{ }(p / 2)$.

Proof. $\quad N(A)=2$. From Lemma 3 , there exist ideals $B, C$ with properties 1 to 5 . Set $b=N(B), c=N(C)$. As $A B C$ is principal there exist 
integers $y, z$ satisfying

$$
N(A B C)=2 b c=y^{2}+2 p z^{2} .
$$

Thus $2 \mid y$. Let $y=2 x$, so that (12) gives

$$
b c=2 x^{2}+p z^{2} .
$$

$A \nmid B C$, so $2 \nmid b c$ and $z$ is odd. Further

$$
b c=N(B C)<-D / 3=8 p / 3<4 p,
$$

so, from (14), $z^{2}=1$, and (13) becomes

$$
b c=2 x^{2}+p=f(x) .
$$

From (15), $x^{2}=(b c-p) / 2<5 p / 6$, so, for $p>3, x<\sqrt{ }(5 p / 6)<(p-1) / \sqrt{ } 2$. Further for $p=3$, as $x$ is integral $x<\sqrt{ }(5 / 2) \Rightarrow x \leqq 1<\sqrt{ } 2=(p-1) / \sqrt{ } 2$. Hence

$$
x<(p-1) / \sqrt{ } 2,
$$

so, by Lemma $1, f(x)$ is composite for some integer $x$ in the interval $0 \leqq x<\sqrt{ }(p / 2)$.

LEMMA 5. In fields of Type II, $h>2 \Rightarrow f(x)$ is composite for some integer $x$ in the interval $0 \leqq x<(\sqrt{ } p-1) / 2$.

Proof. As above we can find $b=N(B), c=N(C)$ and integers $y, z$ so that

$$
N(A B C)=2 b c=y^{2}+p z^{2} .
$$

From Lemma 3, $2 \nmid b c$, so

$$
2 b c \equiv 2 \equiv y^{2}+z^{2}(\bmod 4),
$$

and hence both $y$ and $z$ are odd. Putting $y=2 x+1$ we obtain

$$
b c=2 x^{2}+2 x+\left(1+p z^{2}\right) / 2,
$$

and further

$$
b c=N(B C)<-D / 3=4 p / 3<2 p .
$$

Thus $z^{2}=1$, and (19) becomes

$$
b c=2 x^{2}+2 x+(p+1) / 2=f(x) .
$$

Also, from (20), $2 x^{2}+2 x+(p+1) / 2<4 p / 3$, so $\left(x+\frac{1}{2}\right)^{2}<5 p / 12 \leqq p^{2} / 12 \leqq$ $p^{2} / 8$ (as $p \geqq 5$ ). Hence

$$
0 \leqq x<(p-\sqrt{ } 2) / 2 \sqrt{ } 2 .
$$


However, from Lemma $2, f(x)$ is composite for some integer $x$ in the interval $0 \leqq x<(\sqrt{ } p-1) / 2$.

Lemma 6. In the fields of Type III, $h>2 \Rightarrow f(x)$ is composite for some integer $x$ in the interval $0 \leqq x<\sqrt{ }(-D / 12)-\frac{1}{2}$.

Proof. We may assume $p q>16$, since $Q(\sqrt{ }-p q)$ has class number greater than two. $N(A)=p$. As above we can find $b=N(B), c=N(C)$ and integers $y, z, y \equiv z(\bmod 2)$ so that

$$
N(A B C)=p b c=\left(y^{2}+p q z^{2}\right) / 4,
$$

i.e.,

$$
4 p b c=y^{2}+p q z^{2} .
$$

If $b=2$, then $(2 \mid p q)=1$, so $p q \equiv 7(\bmod 8)$, and hence $p+q \equiv 0(\bmod 8)$. Thus, since $p q>16$, we find that $f(0)=(p+q) / 4$ is properly divisible by 2 and hence composite.

For $b>2$, from (24), $p \mid y$, so let $y=p v$, so that (24) becomes

$$
4 b c=p v^{2}+q z^{2} .
$$

As $A \nmid B C, p \nmid b c$, and hence $z \neq 0$. Also if $v=0$, then $q \mid b c$. However $b$, $c<\sqrt{ }(p q / 3)<q$ so $q \nmid b, c$ and as $q$ is prime $q \nmid b c$. Hence $v \neq 0 . b, p$ are primes, $(b, p)=1$, so $b|z \Rightarrow b| v$, so, from (25),

$$
\begin{aligned}
b \mid z & \Rightarrow 4 c>(p+q) b>2(p+q) \\
& \Rightarrow 4 c^{2}>p^{2}+q^{2}+2 p q>2 p q \\
& \Rightarrow c>\sqrt{ }(p q / 2) .
\end{aligned}
$$

However as $c<\sqrt{ }(p q / 3), b \nmid z$. Thus $z \not \equiv 0(\bmod b)$, so there exists an inverse $z^{\prime}$ of $z(\bmod b)$. From $(25)$ we obtain

$$
p\left(v z^{\prime}\right)^{2}+q \equiv 0(\bmod b) .
$$

Let $w$ be the least positive residue $(\bmod b)$ of $v z^{\prime}$. As $b$ is odd, one of $w, b-w$ is odd, so let $u$ be that value and hence $0<u<b$. From (26), $p u^{2}+q \equiv 0(\bmod b)$ while also $p u^{2}+q \equiv p+q \equiv 0(\bmod 4)$, so

$$
p u^{2}+q \equiv 0(\bmod 4 b) .
$$

Since $A B C$ is principal, and $C$ not principal, neither is $A B$. Thus $p u^{2}+q \neq$ $4 b$, for otherwise $4 b p=(p u)^{2}+p q$, and $A B=((p u \pm \sqrt{ }-p q) / 2)$. Thus $b$ is a nontrivial divisor of $\left(p u^{2}+q\right) / 4$. As $u$ is odd, let $u=2 x+1$ so that

$$
\left(p u^{2}+q\right) / 4=f(x)
$$


which has a proper prime divisor $b$, so is composite. Now

$$
0<x=(u-1) / 2<b / 2-\frac{1}{2}<\sqrt{ }(p q / 12)-\frac{1}{2}
$$

so again the lemma holds true.

This now establishes the first half of the theorem. The remainder is established in a final lemma.

LEMMA 7. For each field of Type I, II or III, if $f(x)$ is composite for some integer $x$ in the interval $0 \leqq x<k$, then $h>2$.

Proof. Suppose $f(x)$ is composite with $0 \leqq x<k$, so that $f(x)=b c$, with $b, c>1$, integral and $b$ prime. Now with $a$ as chosen before Lemma 3,

$$
\begin{aligned}
f(x)=b c \Rightarrow a b c & =(2 x)^{2}+d & & \text { for fields of Type I, } \\
& =(2 x+1)^{2}+d & & \text { for fields of Type II, } \\
& =\left((2 x+1)^{2} p^{2}+d\right) / 4 & & \text { for fields of Type III. }
\end{aligned}
$$

For fields of Type I, $x \neq 0$, as $f(0)=p$ is prime, so we find that for all fields $(b c, d)=1$. Hence, from $(30),(-d \mid r)=1$ for all primes $r$ dividing $b c$.

Let $\alpha$ be the algebraic integer $2 x+\sqrt{ }(-d),(2 x+1)+\sqrt{ }(-d)$, or $((2 x+1) p+\sqrt{ }(-d)) / 2$ in the fields of Types I, II and III respectively. Hence, in all fields of Type I, $x<\sqrt{ }(p / 2) \Rightarrow N(\alpha)=(2 x)^{2}+2 p<4 p$, i.e.,

$$
N(\alpha)<2 d \text {. }
$$

Similarly, in fields of Type II, $x<(\sqrt{ } p-1) / 2 \Rightarrow N(\alpha)=(2 x+1)^{2}+p<$ $2 p=2 d$, i.e.,

$$
N(\alpha)<2 d
$$

For fields of Type III, $x<\sqrt{ }(p q / 12)-\frac{1}{2} \Rightarrow\left((2 x+1)^{2} p^{2}+p q\right) / 4<p^{3} q / 12+$ $p q / 4<p^{2} q^{2}(1 / 12+1 / 60)$, i.e.,

$$
N(\alpha)<d^{2} / 10 .
$$

Using these three inequalities we now prove that the algebraic integer $\alpha$ has no nontrivial factorisation. As the coefficient of $\sqrt{ }(-d)$ in $\alpha$ is 1 , $\alpha$ cannot be divisible by any nontrivial rational integer. Suppose $\alpha$ does have a nontrivial factorisation in algebraic integers,

$$
\alpha=\beta \gamma
$$

where for $D \equiv 0(\bmod 4), \beta=b_{1}+b_{2} \sqrt{ }(-d), \gamma=c_{1}+c_{2} \sqrt{ }(-d)$, and for $D \equiv 1(\bmod 4), \quad \beta=\left(b_{1}+b_{2} \sqrt{ }(-d)\right) / 2, \quad \gamma=\left(c_{1}+c_{2} \sqrt{ }(-d)\right) / 2$, with $b_{1} \equiv$ $b_{2}(\bmod 2), c_{1} \equiv c_{2}(\bmod 2)$.

If $b_{2}=0, \beta$ would be a rational integer, hence $\beta= \pm 1$. Similarly $c_{2}=0 \Rightarrow \gamma= \pm 1$. Hence for a nontrivial factorisation (34) we require 
$b_{2}, c_{2} \neq 0$. For $D \equiv 0(\bmod 4), N(\alpha)=N(\beta) N(\gamma)=\left(b_{1}^{2}+d b_{2}^{2}\right)\left(c_{1}^{2}+d c_{2}^{2}\right) \geqq d^{2}$, which contradicts equations $(31)$ and $(32)$. For $D \equiv 1(\bmod 4)$,

$$
\begin{aligned}
N(\alpha) & =\left(b_{1}^{2}+d b_{2}^{2}\right) \cdot\left(c_{1}^{2}+d c_{2}^{2}\right) / 16 \\
& =\left(b_{1}^{2} c_{1}^{2}+d\left(b_{1}^{2} c_{2}^{2}+b_{2}^{2} c_{1}^{2}\right)+d^{2} b_{2}^{2} c_{2}^{2}\right) / 16 .
\end{aligned}
$$

However as $N(\alpha)<d^{2} / 10$ by equation (33), we must have $b_{2}^{2} c_{2}^{2}=1, b_{1}^{2} c_{1}^{2}<d^{2}$.

Thus, for fields of Types I or II, $\alpha$ can have no nontrivial factorisation (34), and, for fields of Type III, it can only be of the form

$$
\begin{aligned}
\alpha & =((2 x+1) p+\sqrt{ }(-d)) / 2 \\
& =\left(\left(b_{1}+b_{2} \sqrt{ }(-d)\right) / 2\right) \cdot\left(\left(c_{1}+c_{2} \sqrt{ }(-d)\right) / 2\right),
\end{aligned}
$$

with $b_{2} c_{2}= \pm 1$, and $\left|b_{1} c_{1}\right|<d=p q$. By equating real and imaginary parts in equation (35) we find

$$
2(2 x+1) p=b_{1} c_{1}-b_{2} c_{2} d=b_{1} c_{1}-b_{2} c_{2} p q,
$$

and

$$
2=b_{1} c_{2}+b_{2} c_{1} .
$$

As $2(2 x+1) p>0,\left|b_{1} c_{1}\right|<p q$, and $\left|b_{2} c_{2}\right|=1$, then $b_{2} c_{2}=-1$ for (36) to hold. Suppose $b_{2}=1, c_{2}=-1$; then, by equation (37), $c_{1}=b_{1}+2$, and equation (36) becomes

$$
2(2 x+1) p=b_{1}\left(b_{1}+2\right)+p q .
$$

Hence $p \mid b_{1}$ or $p \mid b_{1}+2$, so $p \leqq \min \left(\left|b_{1}\right|,\left|b_{1}+2\right|\right)$. $b_{1} \neq-1$, so $b_{1}\left(b_{1}+2\right) \geqq 0$, and $p(p-2) \leqq b_{1}\left(b_{1}+2\right)$. Thus it follows from equation (38) that

$$
4 x+2 \geqq p+q-2
$$

and, on squaring,

$$
4(2 x+1)^{2} \geqq p^{2}+q^{2}+2 p q-4 p-4 q+4 .
$$

However as $p \geqq 3, q \geqq 5, p^{2}+q^{2}>4 p+4 q-4$, so

$$
4(2 x+1)^{2}>2 p q \text {. }
$$

Also $x<\sqrt{ }(p q / 12)-\frac{1}{2}$ so $4(2 x+1)^{2}<4 p q / 3$. This contradicts equation (41), so we cannot have a factorisation with $b_{2}=1, c_{2}=-1$. Alternatively $b_{2}=-1, c_{2}=1$ leads to the same contradiction, so the factorisation (34) cannot exist in this case.

Thus in all cases $\alpha$ has no nontrivial factors.

Let $A$ be the ambiguous ideal above. As $(-d \mid r)=1$ for all prime divisors of $b c$, there exist ideals $B, C$ with $N(B)=b, N(C)=c$, such that $A B C=(\alpha)$. 
Now $\alpha$ has no nontrivial divisors, so $A B C$ has no principal ideal divisors, and in particular none of $A, B$ and $A B$ can be principal. Thus as $A^{2}$ is principal, $A$ cannot be in the same class as $B$, so the number of classes $h>2$.

\section{REFERENCE}

1. H. Cohn, A second course in number theory, Wiley, New York, 1962. MR 24 \#A3115.

Department of Mathematics, University of New England, New South Wales, Australia

Department of Mathematics, Massey University, Palmerston North, New ZEALAND 